\title{
Chaos surviving machinery
}

\begin{abstract}
Usually the machinery used in engineering applications, especially those implicating cyclic processes make use of oscillating systems that include components vibrating each one with a particular frequency, in a medium with continuously changing muffling, temperature, etc. As a consequence these vibrations may eventually break the harmony of the common oscillation and experiment chaos. Hence some Chaos notions are critical in this type of applications. This work reports the results of a chaos research in a very frequently applied oscillating system. It is found that chaos is an eventual occurrence. After the system undergoes chaos, it returns to its oscillating stage under the conditions imposed by the medium (muffling, temperature, frequency, etc.) at the moment of leaving chaos. This leads to considering the eventual design of chaos surviving machinery
\end{abstract}

Keywords: chaos, computer simulation, non-linear systems, oscillations,

Maquinaria que sobrevive al CaOs

\section{RESUMEN}

Generalmente la maquinaria usada en aplicaciones de ingeniería, especialmente aquellas que involucran procesos cíclicos, constan de sistemas oscilantes que incluyen componentes que vibran cada uno con una frecuencia particular, en un medio donde continuamente varían la amortiguación, la temperatura, etc.. En consecuencia estas vibraciones podrían eventualmente romper la armonía de la vibración común y experimentar Caos. Por lo tanto, algunas nociones sobre Caos son importantes en este tipo de aplicaciones. En este trabajo se reportan los resultados de una investigación de Caos en un sistema oscilante muy frecuentemente usado. Se encuentra que el Caos es eventual, después de experimentarlo, el sistema retorna a su estado oscilante bajo las condiciones que le impone el medio (temperatura, fricción, frecuencia, etc.) en el momento de abandonar el caos. Esto conduce a considerar la eventualidad de diseñar máquinas que sobreviven al caos.

Palabras clave: caos, oscilaciones, simulación computarizada, sistema no-lineal,

\section{INTRODUCCIÓN}

Generally linear processes are easy-to-deal-with idealizations used in the classroom; in real life processes are mostly nonlinear. Chaos is a phenomenon eventually observed in nonlinear oscillating systems where there are at least two competing oscillating frequencies. Chaos has been encountered in physics, engineering, and economics $[1,2,3]$.

The study of Chaos must be necessarily carried out by means of numerical computer simulation of mathematical models. In the simulation one or more parameters -like time, dumping, temperature, etc- are allowed to vary, sometimes until reaching extreme values. In real life the study of chaos has as its main limitation the fact that real life systems are not constructed to undergo chaos and they collapse very quickly, if not before reaching chaos, a little later after chaos sets in.

The purpose of the investigation here reported has been to find out what happens beyond chaos in a non-linear forced and damped oscillator in which the transition to chaos had previously been studied in up to one million time-steps $[4,5,6]$.

It is known that chaos may eventually show up in oscillating systems with at least two competing frequencies. This research tries to find out whether once a system enters.

Chaos, it remains in that chaotic stage without ever finishing it, or if the chaotic status is eventually abandoned and what happens once chaos finishes, if it ever does.

A basic model of a centrifugal pendulum vibration absorber [7] used in land vehicles and in other engineering applications is shown in fig 1 . This is an oscillating system with two competing frequencies. The spring oscillating along the horizontal has its own frequency, while the pendulum attached to the spring and oscillating on a vertical plane has also its particular frequency. The resistance of the air is different for the spring and for the pendulum and affects their oscillation frequencies. Eventually the frequencies of spring and pendulum enter into conflict and the system becomes chaotic. This kind of vibration absorber is being also used in modern buildings as a means of dampening the vibrations due to earthquakes.

* VirtualDynamicsSoft: Science \& Engineering Virtual Labs - Physics Faculty at UNMSM. E-mail: Director@VirtualDynamics.Org --- www.VirtualDynamicsSoft.com 


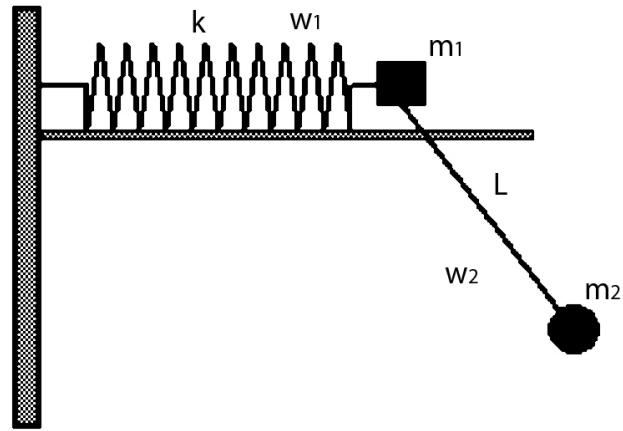

Figure 1. A mechanical system whith two oscillating frequencies $\omega_{1}$ and $\omega_{1}$. This is a system prone to chaos: eventually the particular frequencies of spring and pendulum break up their harmony and the system becomes chaotic.

When for some reason it is necessary to maintain the oscillations in the system of Fig. 1, it must be connected to an external vibrator, which adds a third frequency to the system, and which also increases its likeliness of unleashing chaos.

\section{MACHINERY COLLAPSE DUE TO CHAOS}

When an vibrating system begins to experiment chaos, its vibrations (oscillations) alternate between two periods (frequency $f$ is the inverse of period T: Tf $=1$ ), then each of these split and appear four periods, and so on until chaos sets in. Machines made of two or more oscillators, like those represented by Fig. 1 and 2 , are built to harmoniously oscillate with a common single frequency (period), they are not prepared to oscillate under a chaotic regime, for this reason they collapse a little after a chaotic regime arises.

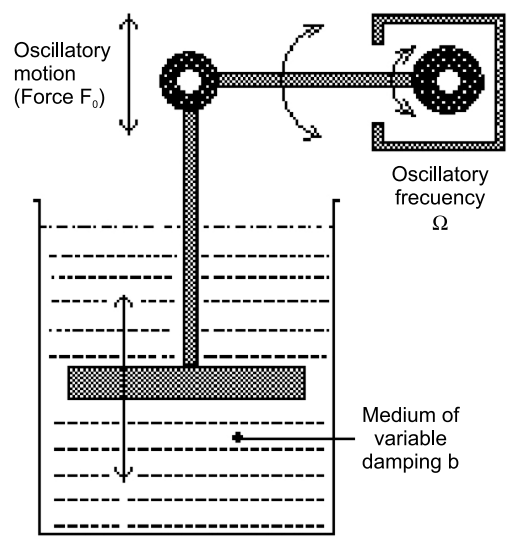

Figure 2. Forced oscillations in a variable damping medium.

\section{USING COMPUTER SIMULATION TO STUDY CHAOS}

Since manmade devices are not prepared to endure chaos, its study must make use of computer simulation. In this way it is clear that chaos investigation depends strongly on computational resources and on computer programming techniques. Some years ago it was impossible to execute a one million time steps computer simulation. Back then the visualization in real time of an oscillating object on monitor screen, and performing the corresponding calculations in parallel was no more than a cherished dream. Nowadays this is achieved almost regularly.

\section{PREVIOUS INVESTIGATION}

Making use of the same mathematical model used in this investigation, the author has previously investigated the transition to chaos in up to one million time steps $[4,5,6]$. The investigation being reported here is based on computer simulations in up to six million time steps, and it has been found that after a chaotic event the system returns to its normal oscillatory regime and continues its oscillation as if it were just a forced oscillator.

\section{THE RESEARCH}

The investigation here reported has been performed using a very common combination of oscillators, whose physical model is shown in Fig. 2. This system is mathematically modeled [6] by the second order differential equation

$$
\frac{d^{2} x}{d t^{2}}+2 b \frac{d x}{d t}+\omega_{o}^{2} \operatorname{Sin} x=F_{o} \operatorname{Sin} \Omega t
$$

and it represents a non-linear damped and forced oscillator, submitted to an external oscillating force $F_{\text {o }}$ whose frequency is $\Omega$. The oscillator whose natural frequency is is immersed in a medium of variable damping $b$. The amplitude of the oscillations is $x$, and $t$ is the evolving time. In order to get a variable damping, let's say an increasing one, assume that the medium becomes thicker as time elapses, this may be achieved in real life with a gelatinous material. The opposite effect is achieved with oil, which becomes thinner as temperature raises. 


\section{NUMERICALLY SOLVING THE DIFFERENTIAL EQUATION}

In this investigation the differential equation modeling the oscillator is numerically solved by means of the Runge-Kutta technique, using a computer program developed by the author of this paper. This software also detects other chaotic events in the system under study.

Interested readers not inclined to computer programming may use software packages like Mathlab, Mathematica, etc. which are available on the market.

\section{RESULTS}

In this research in order to simulate a variable damping medium, the damping factor $b$ was kept constant and the applied force $F_{0}$ was gradually increased.

Fig. 3 shows the results of the investigation for one million time steps, $[4,5,6]$ it is a plotting of amplitude versus time, and as it may be seen, the behavior of the system is unknown beyond the first million time steps.

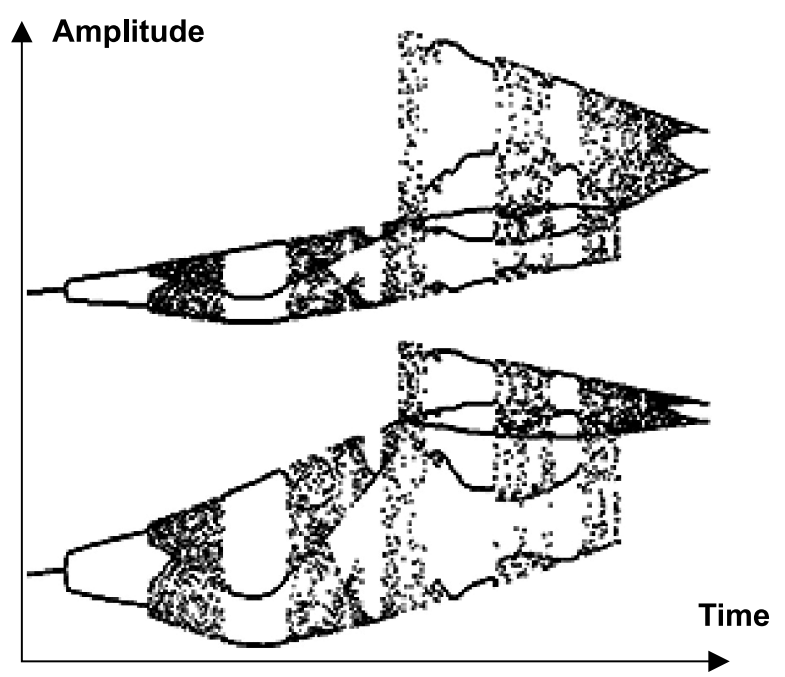

Figure 3. Bifurcation cascade. One-million time steps What happens once Chaos finishes?

Figure 4 displays the results of a simulation along six million time steps, as it can be seen, the system returns to its damped and forced oscillating behavior.

Figure 5 shows the Return Map, which is a plotting of the oscillation amplitude at time $t+1$ versus the amplitude one step before, at time $t$. As it can be seen, it is impossible to foresee the oscillation amplitude at time $t+1$, having as a reference the amplitude at time $t$. Notice that if all oscillations had the same amplitude, this plotting would simply be a single dot.

\section{DISCUSSION}

A computer-simulation based research on the chaotic behavior of a non-linearly forced and damped oscillator has been carried out. In a previous research with the same mathematical model, the evolution through one million time-steps was investigated.

This time the aim of the research was to observe what happened well beyond one million time steps and hence this research went throughout six million time steps.

It has been found that the system enters chaotic behavior by means of period bifurcations and then abandons this stage with the same smoothness with which it adopted this comportment; this is, by means of inverse period bifurcations.

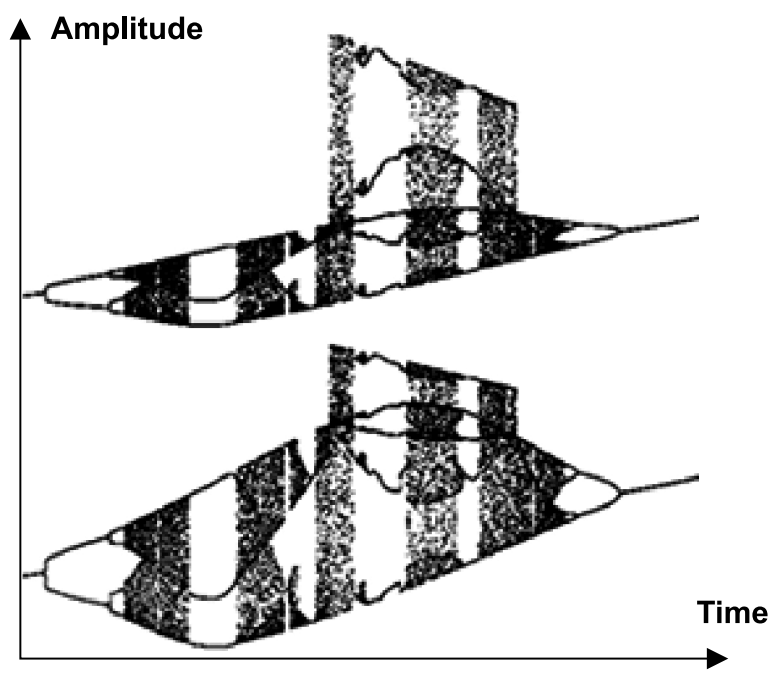

Figure 4. Period bifurcations cascade. Six million time steps. After chaos the system returns to its oscillations, this means that chaos is not forever, it is just an event.

Hence, it has been encountered that chaos -at least in the system studied- is an event lasting some time, after which the system returns gradually to normality, this is, the system returns to its forced oscillations, notwithstanding the conditions that lead the system to chaos have not disappeared, they are still maintained. 
Since the system was always submitted to an applied external oscillating force which was gradually incremented, once the chaotic event finishes, the amplitudes of the oscillations are increasing as if the chaotic event had no influence at all. With the aim of clarifying this point, note that if the oscillating system had not underwent chaos, the amplitude of its oscillations would had simply increased due to the external increasing applied force. Since the same behavior is being observed after chaos, it might be concluded that at first sight chaos had no influence in the post-chaos behavior of the system.

The developed software allowed the detection of three additional chaotic events in the system under study, and it has been observed that all of them display the same general behavior.

\section{SURVIVING-CHAOS MACHINERY}

Automobiles are provided with robustness enough so that they can overcome the potholes on the road, at least those no so big. In the same way, machines may be designed so that they are able to survive chaotic events.

If chaos is an eventual behavior of some oscillating systems, and these sooner or later return to normality, then there is the possibility of conceiving surviving-chaos machines, something like life after chaos for machinery. Hence a machine undergoing chaos may eventually finish it and return to its normal behavior.

Consequently, the problem with those machines that collapse when undergoing chaos is that these are systems lacking robustness enough to transit successfully throughout chaos. Then disregarding costs, if at the time of devising machines those are enabled with robustness enough to survive chaos, the result will be something tantamount to selfrepairing machines.

\section{CHAOS OF THE HEART}

Devising a mathematical model for the oscillations of the heart is not easy, however the knowledge acquired from chaos simulations may help to understand the behavior of our hearts.

The heart consists of a huge number of muscular fibers acting as oscillators. In a healthy heart all its fibers oscillate simultaneously and with the same frequency, alternating between diastole (expansion) and systole (contraction). If due to some reason some of these oscillators change frequency, arrhythmia appears and the system becomes prone to chaos. Cardiac fibrillation may be equivalent to transition to chaos.

Making a parallel of the behavior of the heart with the model reported in this paper, it may be said that an increasing viscosity of the blood and the widening of the walls of the veins, introduce some kind of damping for the oscillations of the heart. Additionally, the heart may be regarded as a forced oscillator because the brain sends electric signals forcing the former to oscillate at a rhythm dictated by the latter.

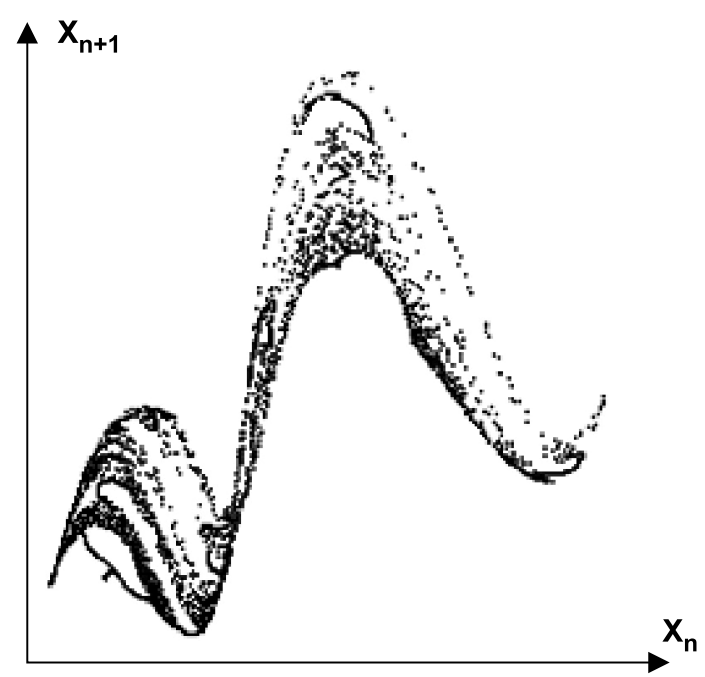

Fig. 5. Return map.

It is very well known that some people undergoing a heart attack survive it without any help. Perhaps the explanation from the point of view of Chaos is that those survivors possess a rather robust heart which allows them to go through a rather mild chaotic event.

\section{CONCLUSIONS}

1. By means of a Runge-Kutta numerical computer simulation in software developed by this author, Chaos has been investigated in a damped and non-linear forced oscillator, throughout six million time steps.

2. It has been found that Chaos is not a permanent condition, it is rather an episode in the behavior of the oscillator, this is, an event.

3. The chaotic event finishes with the system gradually returning to its status of forced oscillator. 
4. The system enters chaos by means of period bifurcations and abandons it by means of the opposite behavior; this is, by period duplications.

5. In the mathematical model used for the simulation the oscillator is subject to an increasing external oscillating force, hence the oscillation amplitudes increase gradually, and this is observed once the system abandons chaos.

\section{REFERENCES}

[1] Hilborn R.C., Chaos and Nonlinear Dynamics, Oxford Univ. Press. Preprint.

[2] Zaslavsky, G.M.(1999) Chaotic Dynamics and the Origin of Statistical Laws, Physics Today, August 1999.
[3] Gonzales-Miranda, J.M. (2004) Synchronization and Control of Chaos, an Introduction for Scientists and Engineers. Imperial College Press, London.

[4] Montenegro J.J., Machinery breakdown: a Chaos-Based Explanation. Industrial Data, Vol. 12, No 1, 2009

[5] Montenegro J.J.,Transition to chaos in the damped \& forced non-linear oscillator. Rev. Inv. Fis., UNMSM, Vol. 12, No 1, 2009

[6] Montenegro J.J., A pragmatic introduction to Chaos Theory for engineers, Industrial Data, Vol. 12, No 2, 2009

[7] Balachandran B. - Magrab E., Vibrations, Brooks-Cole Editors, 2004 